Gut, 1972, 13, 1-7

\title{
Experimental venous lesions of the colon
}

\author{
R. W. MARCUSON, J. O. STEWART, AND ADRIAN MARSTON
}

From the Department of Surgical Studies and the Bland Sutton Institute of Pathology, The Middlesex Hospital, London

SUMMARY This experimental work has shown that structural changes in the colon may be produced following inferior mesenteric vein thrombosis. Whilst there are certain radiological similarities, sigmoidoscopic appearances and histological studies show that the pathogenesis of venous lesions differs from that of arterial lesions. The possible relationship of these experiments to clinical lesions is discussed.

\section{Methods}

Adult mongrel bitches were used for this study. As far as possible they were admitted to the laboratory one week before surgery for stabilization on a standard dog diet. No bowel preparation was carried out, and the dogs were starved overnight preceding surgery. Baseline leucocyte counts and alkaline phosphatase estimations were carried out as described below.

\section{SURGICAL PROCEDURES}

Anaesthesia was induced with intravenous pentobarbitone $30 \mathrm{mg} / \mathrm{kg}$ which was supplemented as necessary. The abdomen was entered through a midline incision and the normality of the abdominal contents was verified. A diagram of the anatomy of the venous drainage of the colon is shown in Figure 1. Only minor individual variations in this pattern were found. Three ligatures were applied as shownone to the main inferior mesenteric vein and two to the marginal vein (proximally and distally) -and a $15 \mathrm{~cm}$ length of vein was thereby isolated. Simple ligations were carried out in five dogs. In a second series of 13 dogs, $200 \mathrm{NIH}$ units of bovine thrombin in $4 \mathrm{ml}$ of water was injected between the ligatures to produce a solid thrombus using the method described by Polk (1966) in his work on experimental superior mesenteric vein thrombosis. Fast injection of the solution was required and some technical difficulties were encountered because of the very rapid development of high pressure within the thinwalled vein following the application of the ligatures. The abdomen was closed in layers. Intravenous fluids were given to three dogs.

\footnotetext{
Received for publication 16 November 1971.
}

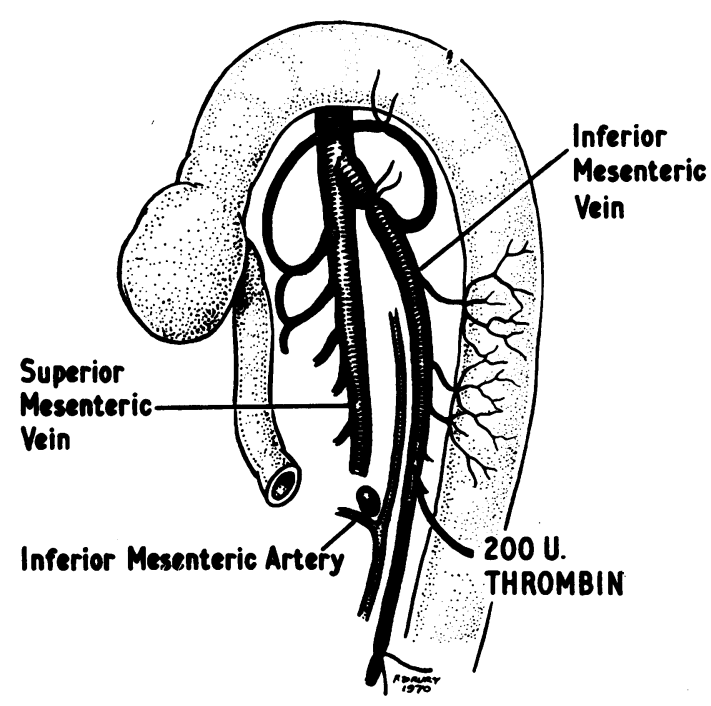

Fig. 1 Diagram to show the anatomy of the venous drainage of the dog colon, the sites of the ligatures, and the site of the thrombin injection.

\section{SIGMOIDOSCOPIC ASSESSMENT AND SEQUELAE}

Immediate postoperative sigmoidioscopy was undertaken to assess the severity of the lesions produced. The examination was repeated without anaesthesia on several subsequent occasions to assess progress of the lesions. Dogs were divided into three groups based on the severity of their lesions as observed during the immediate postoperative sigmoidoscopy. Animals from each group were sacrificed at intervals from one day to six weeks and the colon was examined with particular reference to the correct 
siting of the original ligatures and the development of new venous pathways. The colon and rectum were then removed by abdomino-perineal resection. The specimens were opened and washed clean with cold physiological saline, pinned out, and fixed in formalsaline in preparation for histological examination.

\section{RADIOLOGY}

Serial barium enemas were performed under general anaesthesia using the method described by Chapman in Marston et al (1969). Several dogs were examined on the first postoperative day. Other examinations were performed when indicated by the sigmoidoscopic findings.

\section{LEUCOCYTE COUNTS}

Blood was taken for total leucocyte counts immediately preoperatively, and on the first and second postoperative days. The counts were made using a Coulter counter.

\section{ALKALINE PHOSPHATASE ESTIMATIONS}

The total serum alkaline phosphatase was measured by standard methods using an autoanalyzer. The intestinal alkaline phosphatase fraction was assayed with a modification of the L-phenylalanine inhibition method of Fishman, Green, and Inglis (1963), and by Marcuson and Tomlinson (1971).

Sera for these examinations were obtained preoperatively and on the first two postoperative days. Two dogs served as controls for those animals described above as having received thrombin in the inferior mesenteric vein. These control animals were anaesthetized and given the same dose of thrombin (200 NIH units) by systemic intravenous injection. Samples of blood for alkaline phosphatase estimation were taken before and 24 hours after thrombin administration.

\section{Results}

CHANGES AT OPERATION

After the ligatures to isolate the inferior mesenteric vein were tied the main vein and vasa recta became tense and tortuous. These changes were more marked following thrombin injection and 'thrombin thrombi' were usually formed within five minutes. Normal pulsations were initially visible in the arterial vasa recta but gradually became reduced in amplitude and, in most cases, disappeared within 15 minutes. By this time the mesentery had become oedematous and congested and the colon was no longer transilluminable. Thickening of the colon was obvious on palpation and, in some cases, marked spasm occurrod. Dilated lymphatic channels became prominent on the surface of the colon and adjacent to the inferior mesenteric artery in the colonic mesentery.

\section{SIGMOIDOSCOPY}

Immediate postoperative sigmoidoscopy revealed the extent of the lesions produced. The lesions were classified as severe, moderate, or minimal. Severe venous lesions consisted of gross submucosal congestion with dark blood and mucosal oedema. Plasmalike fluid wept from the surface and in three cases there was bleeding. Moderate lesions consisted of an area of confluent petechial haemorrhages with some mucosal oedema. Minimal lesions consisted of scattered petechial haemorrhages. At immediate postoperative sigmoidoscopic assessment there were eight severe, three moderate, and seven minimal lesions. Three further groups emerged on follow-up examination. The first of these consisted of 11 dogs with lesions which underwent rapid resolution during the first few postoperative days. Three of these initially had severe lesions, three had moderate lesions, and five had minimal lesions. The second group consisted of three dogs all with initially severe lesions which progressed. The mucosa gradually darkened and the dogs died of peritonitis following gangrene of the colon on the fourth or fifth postoperative day. The third group was made up of only one dog in which the initially severe lesion neither progressed nor completely resolved. By the fourteenth postoperative day it had developed a smooth regular stricture about $10 \mathrm{~cm}$ above the anal verge.

\section{LEUCOCYTE COUNTS}

The mean preoperative leucocyte count for the whole group was $11,200 \pm 6,400 / \mathrm{c} \mathrm{mm}$. There was a brisk leucocytosis in all groups and the extent of the rise was related to the severity of the lesion. Table I shows the mean and the standard deviation of the leucocyte counts for each group of dogs, preoperatively and on the first postoperative day, the groups being those classified by immediate postoperative sigmoidoscopic assessment as described above.

\begin{tabular}{llll}
\hline Severity of Lesion & No. of Dogs & \multicolumn{2}{l}{$\begin{array}{l}\text { Leucocyte Count } \times \\
10^{-3} / \mathrm{c} \mathrm{mm}\end{array}$} \\
\hline & & Day 0 & Day 1 \\
\cline { 2 - 3 } & & $11 \cdot 8 \pm 5 \cdot 4$ & $33 \cdot 7 \pm 10 \cdot 0$ \\
Severe & 6 & $11 \cdot 3$ & $21 \cdot 0$ \\
Moderate & 2 & $10 \cdot 4 \pm 8 \cdot 0$ & $20 \cdot 6 \pm 8 \cdot 3$ \\
Minimal & 6 &
\end{tabular}

Table I Leucocyte counts on days 0 and 1

Statistical comparison of the severe and minimal lesion groups shows that on day 0 the groups are 
similar $(0.8>\mathrm{P}>0.7)$, and on day 1 the difference between the two groups is highly significant $(P<$ 0.05).

\section{ALKALINE PHOSPHATASE ESTIMATIONS}

The mean preoperative alkaline phosphatase level for the group was 5.3 \pm 2.8 King Armstrong (KA) units $/ 100 \mathrm{ml}$. Table II shows the preoperative and first day postoperative levels of the groups classified as before.

\begin{tabular}{llll}
\hline Severity of Lesion & No. of Dogs & \multicolumn{2}{l}{$\begin{array}{l}\text { KA units } / 100 \mathrm{ml} \\
\text { Mean and SD }\end{array}$} \\
\hline & & Day O & Day 1 \\
\cline { 2 - 3 } & & $5 \cdot 9 \pm 2 \cdot 4$ & $14 \cdot 7 \pm 6.3$ \\
Severe & 6 & 4.8 & $10 \cdot 1$ \\
Moderate & 3 & $5.2 \pm 3.9$ & $12.6 \pm 5 \cdot 7$ \\
Minimal & 5 &
\end{tabular}

Table II Alkaline phosphatase levels on days 0 and 1

Although there is an obvious rise in the level of alkaline phosphatase activity, statistical analysis shows that the severe and minimal lesion groups are essentially similar both preoperatively $(0.98>\mathrm{P}>$ $0.97)$ and postoperatively $(0.70>P>0.60)$.

The two control animals which received systemic thrombin also had a rise in alkaline phosphatase activity $(5.7$ to 7.0 and 2.6 to $3.2 \mathrm{KA}$ units $/ 100 \mathrm{ml}$ ), but this was considerably less than that observed in the experimental groups.

The special assay for the intestinal component of alkaline phosphatase showed preoperative levels in all the dogs which were less than $10 \%$ of the total activity. There was no postoperative rise in the intestinal component.

\section{RADIOLOGY}

Filled, drained, and air-contrast films were taken during each barium enema examination. The normal canine colon is uniformly tubular, lacks haustral folds, and has a featureless mucosa (Fig. 2).

In dogs initially noted to have severe lesions, there was marked 'thumbprinting' over a long length of colon and frequently there was associated spasm of the bowel (Fig. 3). In one case, a smooth stricture was demonstrated two weeks postoperatively (Fig. 4). A return to normality was demonstrated in longterm survivors.

Less marked 'thumbprinting' than that seen in dogs with severe lesions was demonstrated in two cases with moderate lesions. In dogs with minimal lesions minor changes of spasm and mucosal irregularity were occasionally observed.

\section{PATHOLOGY}

In several of the dogs sacrificed after the fourth postoperative week, an extensive system of new venous channels had developed (Fig. 5). The veins crossed the mesocolon to the retroperitoneal structures, in particular towards the perirenal tissues.

Seven specimens were obtained at intervals from one day to six weeks from animals reported to have minimal lesions on the first day. Macroscopical findings were minor, amounting at the most to scattered petechial haemorrhages which showed through the mucosal surface. Histological examina-

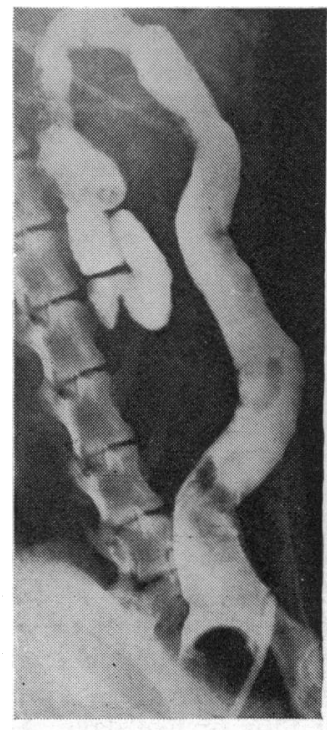

a

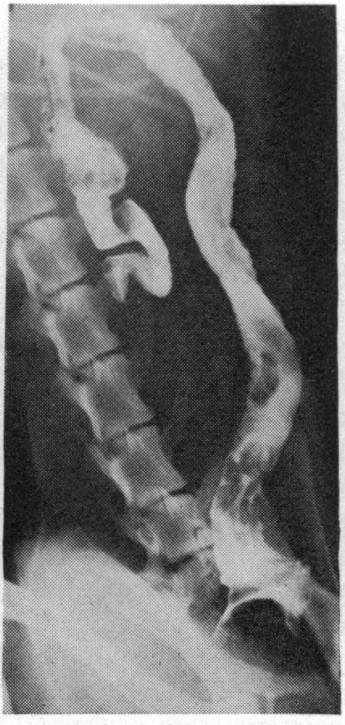

b

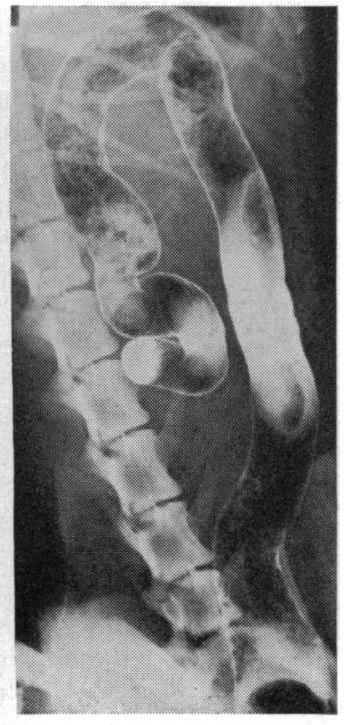

c
Fig. 2 Normal barium enema appearances: (a) colon filled with barium; (b) after drainage; (c) air contrast film. 


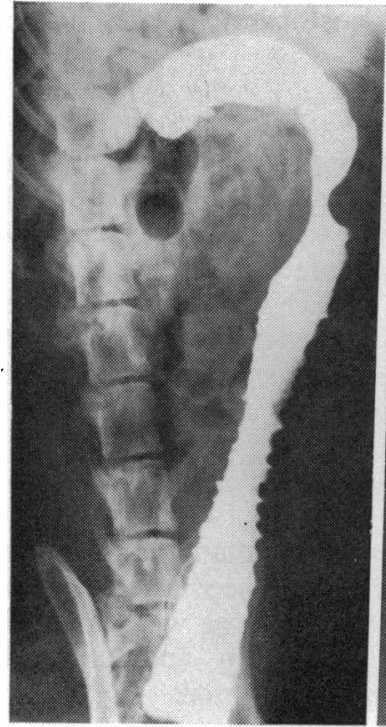

a

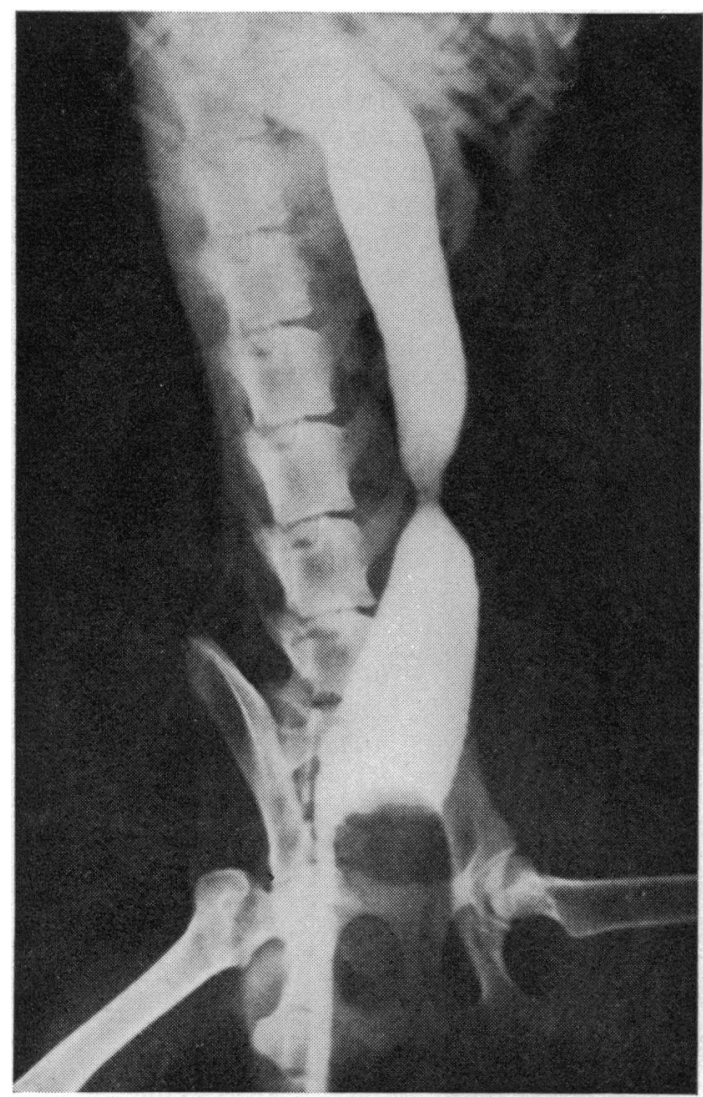

Fig. 4 Barium enema 14 days after a severe lesion showing a persistent stricture. Filled film only.

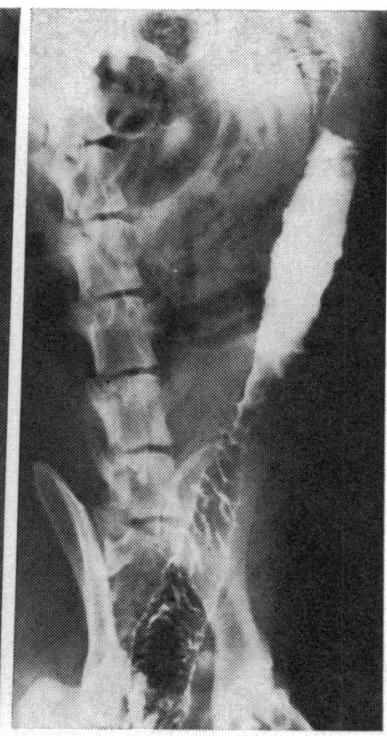

Fig. 3 Barium enema 24 hours after a severe lesion to show extensive thumbprinting: (a) filled film; (b) drained film, and $(c)$ air contrast. tion revealed no significant variation from the normal except for one specimen obtained at three weeks, which showed moderate haemosiderin deposition in the regional mesenteric lymph node.

Three specimens were obtained from animals reported to have moderate lesions on the first day. One of these, examined on the fourth day, showed mucosal discolouration and thickening of the bowel wall. Histological examination revealed an intact mucosa with extravasation of red blood cells into the submucosa and mesentery. There were a moderate number of haemosiderin-containing macrophages in the submucosa, the muscle, and the regional mesenteric lymph node. In the colon examined at two weeks, the mucosa was also intact. There were numerous haemosiderin-containing macrophages in the mesentery and the mesenteric lymph node showed dilated sinuses which were filled with these macrophages. One colon was examined at six weeks and both macroscopical and histological features were normal.

Eight specimens were examined from animals with severe lesions at one day. One of these was obtained 18 hours postoperatively and the colon showed marked congestion of the whole thickness of the bowel wall and of the mesentery (Fig. 6). Histology confirmed the occurrence of congestion with extensive extravasation of blood at all sites. The mucosa was intact. The lymphatics were prominent both in the submucosa and the mesentery. The mesenteric lymph node had marked dilatation of the sinuses which were filled with red blood cells clustered around macrophages (Fig. 7). Three specimens were examined at four or five days. All 


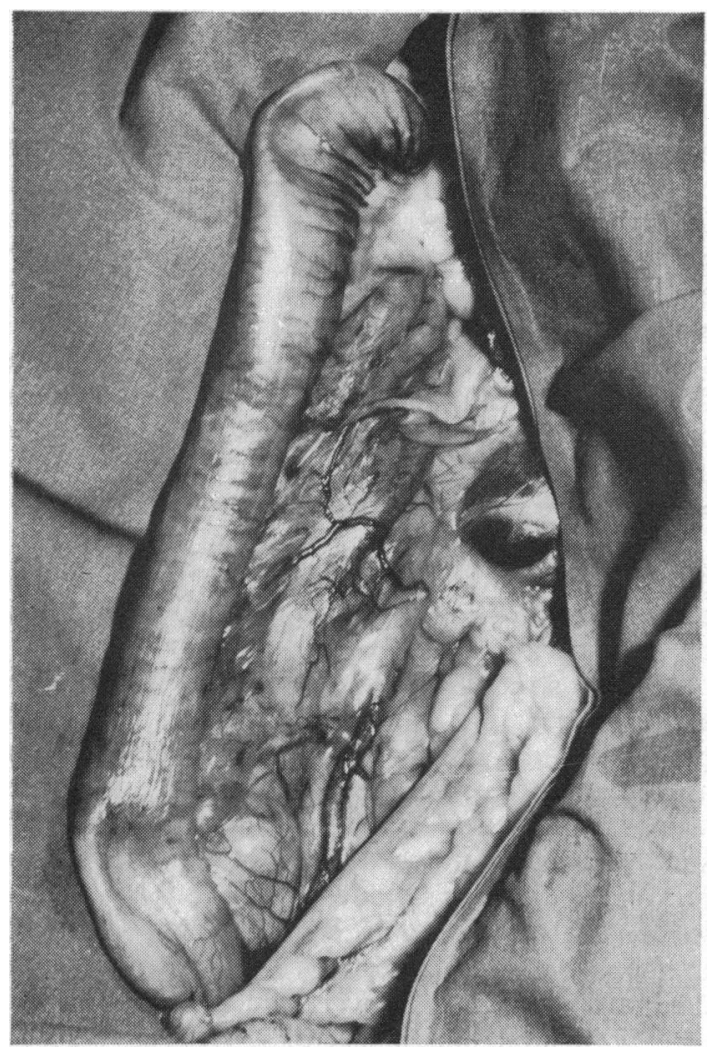

Fig. 5

Fig. 5 Postmortem appearances 42 days after a severe lesion showing extensive venous collaterals.

Fig. 6 Severe lesions at 18 hours, showing marked mucosal congestion and thickening of the mesentery.

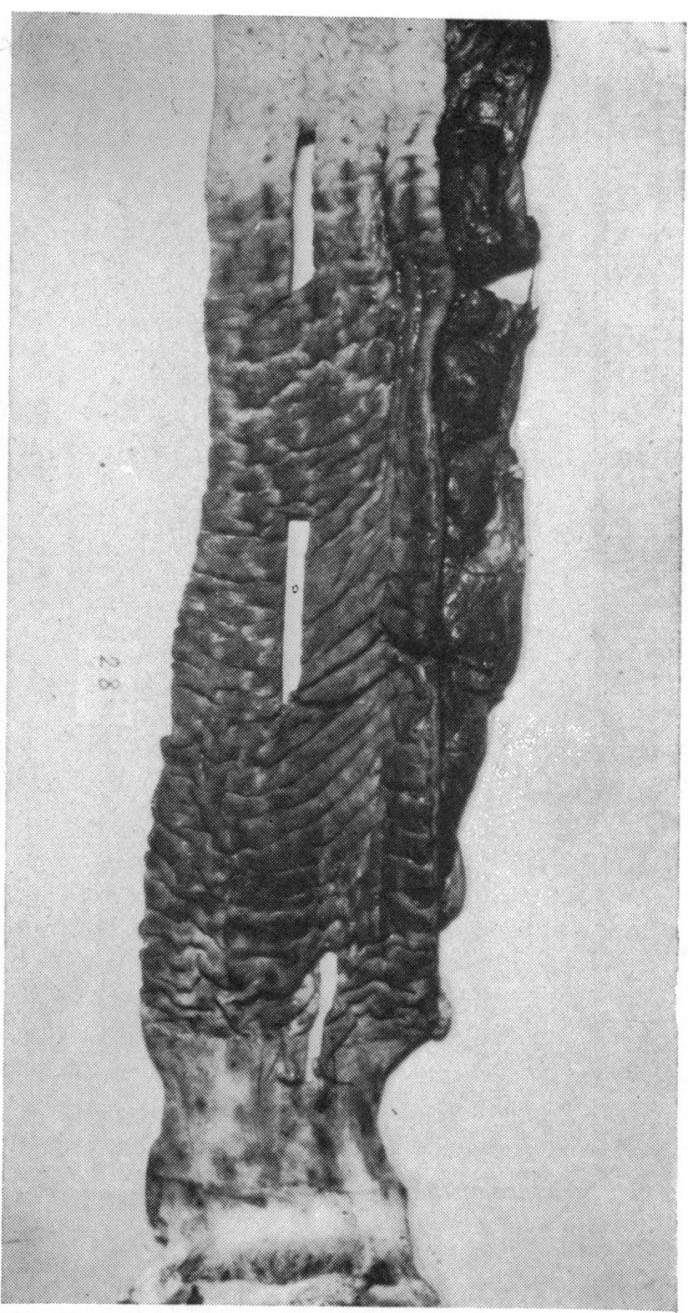

Fig. 6
Fig. 7 Transverse section of colon and mesentery from the same case as Figure 6. Section shows widening of the submucosa and gross thickening of the mesentery. Diffuse patches are extravasated red cells; note thrombus in vein, patency of artery, and gross dilatation of lymph node. Whole mount $\times 7$. Haematoxylin and eosin. 


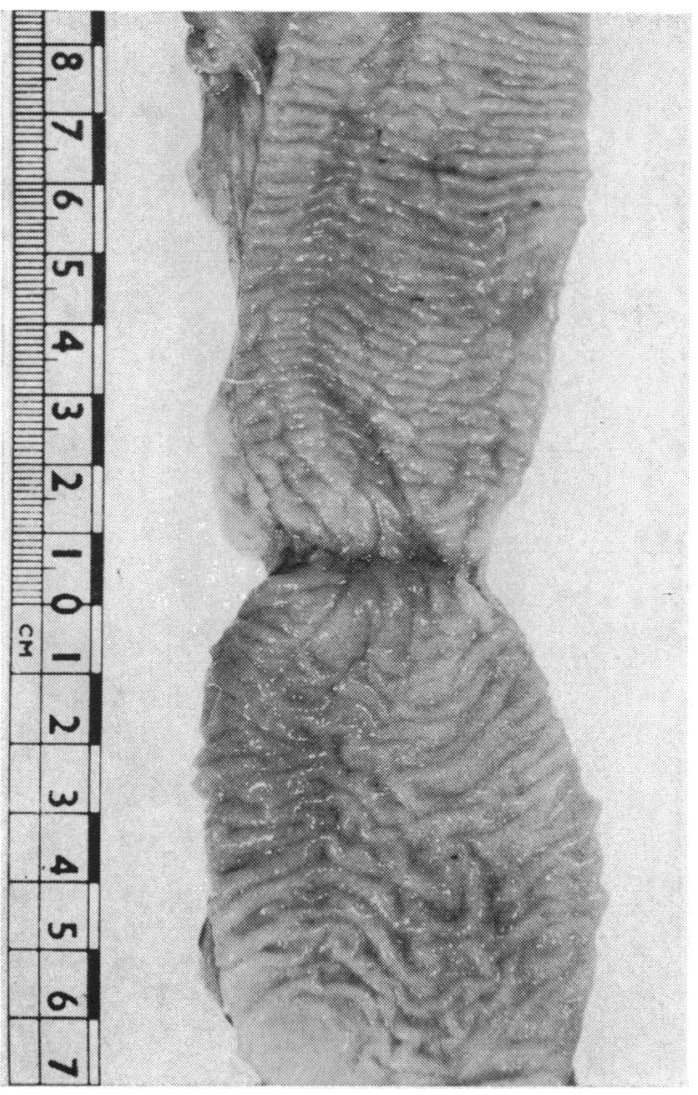

Fig. 8 Stricture of the colon at two weeks.

of these showed extensive areas of full-thickness gangrene with perforation. The demarcation of the necrotic lesion was fairly clear-cut, the adjacent bowel being congested with intact overlying mucosa. Bacterial culture from one specimen demonstrated Clostridium welchii and Gram's stain of histological sections revealed characteristic organisms in the necrotic areas and adjacent submucosa in two of the three specimens. An inflammatory cell infiltration was not prominent. One specimen examined at two weeks showed a narrow annular sticture (Fig. 8). Ulceration and necrosis down to the inner layers of the circular muscle were apparent on histology. There was mucosal regeneration at the edges of the ulcer and there was increased collagen in the submucosa. All three specimens examined at six weeks appeared macroscopically normal. Histology showed normal architecture, but there was a great excess of haemosiderin-containing macrophages in the mesenteric lymph nodes. These nodes had lost the prominent dilatation of the sinuses seen in more acute lesions.

\section{Discussion}

No previous work has been reported on the sequelae of experimental inferior mesenteric vein thrombosis. In the first five experiments, it was attempted to produce thrombosis of the vein by the application of ligatures. Thrombosis was never produced although a severe lesion resulted in one dog.

In an attempt to produce severe lesions more frequently, a second series of 13 experiments was carried out in which thrombin was injected into the inferior mesenteric vein to cause local thrombosis. Seven severe lesions were thereby produced. Scott (1970) has shown that the histological appearance of 'thrombin thrombi' is similar to that found in cases of phlebothrombosis in man and states that these thrombi are a relevant experimental tool.

Marked structural changes occurred in the colon after induced inferior mesenteric vein thrombosis but there are important differences between the appearances of experimental arterial and venous lesions. In the acute stages of the severe lesion, both showed congestion and oedema of the submucosa, but mucosal necrosis, which is frequent in arterial lesions, is rare in venous lesions. When it occurs in venous lesions the necrosis appears to be secondary to submucosal necrosis and is usually associated with fatal muscle necrosis. Arterial mucosal lesions show healing from the underlying submucosa.

Evidence of alternative pathways for the return of venous blood and tissue fluid was seen during the operation: there was dilatation of lymphatics and congestion of the mucosa. These features are also prominent in the early histological picture. In later stages, after resolution, evidence of red blood cell sequestration was seen as haemosiderin deposition in the colonic submucosa and in the regional mesenteric lymph node. This last feature was highly characteristic.

As with animals which had undergone arterial ligation (Marston et al, 1969), sigmoidoscopy proved to be a valuable method of examination of the venous lesions and revealed certain differences in the sequelae of the two operative procedures. Immediate postoperative examination demonstrated the considerably more rapid development of the venous lesion and the widespread blue congestion of the mucosa was quite different from the pale mucosal slough which characterized the severe arterial lesion.

It is suggested that, whilst both are manifestations of hypoxia, arterial lesions represent ischaemic hypoxia and venous lesions represent stagnant hypoxia.

The radiological features correlated well with the sigmoidoscopic appearances, particularly in the case 
of severe lesions. The extent of the 'thumbprinting' was notably marked and was frequently more severe than that cobserved following arterial lesions. It was similar to that occurring in ischaemic colitis as first demonstrated by Boley, Schwartz, Lash, and Sternhill (1963).

The extent of the leucocytosis in dogs in this study appeared to be related to the severity of the initial lesion. The most severe lesions, which resulted in death from gangrene of the colon, were all associated with a leucocyte count greater than $40,000 / \mathrm{c} \mathrm{mm}$ in the first two postoperative days.

In the previous work on arterial lesions, the levels of serum glutamic oxalate transaminase (SGOT), serum glutamic pyruvate transaminase (SGPT), lactic dehydrogenase (LDH), and alkaline phosphatase were all measured daily for seven days postoperatively in an attempt to find a diagnostic biochemical test for colonic ischaemia. There was no rise in SGPT and the rises in SGOT and LDH were non-specific. However, there was a significant rise in alkaline phosphatase and the extent of the rise was found to be related to the severity of the lesion. It was, therefore, decided that only alkaline phosphatase should be measured in the present study, and although a definite rise occurred, it was not related to the severity of the lesion and was probably nonspecific. The control experiments in which systemic thrombin was injected show that the rise was not related to thrombin injection itself.

Doubt still remains about the significance of these experimental results and the possible aetiological relationship which might occur between venous thrombosis and ischaemic disease of the colon in man. Kilpatrick et al (1968) reported two cases of ischaemic colitis in women taking oral contraceptives. These showed definite radiological evidence of 'thumbprinting' with early resolution. We have reported three similar cases (Marcuson and Farman, 1971). No surgery was carried out in any of the five cases and, therefore, no material was available for histological examination. We have also seen one case with a colonic stricture in a woman on oral contraceptives. This lesion was resected but the histological features did not suggest that venous thrombosis was an important aetiological factor. It should, however, be pointed out that the histological features of the experimentally produced stricture in this study were indistinguishable from those of a stricture produced in the study of arterial lesions.

There have been four cases reported in the literature of young women taking oral contraceptives who developed superior mesenteric vein thrombosis (Reed and Coon, 1963; Lowry, Orr, and Wade, 1969; Civetta and Kolodny, 1970; Miller, 1971). This is a most unusual disease in this age group and suggests that both superior and inferior mesenteric vein thrombosis may occur in association with oral contraceptive therapy. It supports the interpretation that colonic changes in such patients follow mesenteric vein thrombosis.

We wish to thank Professor R. H. S. Thompson and Mr F. Yeomans of the Courtauld Institute of Biochemistry for permission to use their facilities and for carrying out the alkaline phosphate estimations, and Dr P. J. Crosland-Taylor and the staff of the Bland-Sutton Institute of Pathology for the haematological studies. We also thank Sister M. V. Tomlinson and Miss S. Corston of the Department of $X$-ray Diagnosis for help with the radiological studies, Mr A. C. Catchpole and his staff for the care of the animals, Mr C. Y. Lau for the histological preparations, and $\mathrm{Mr} \mathbf{M}$. Turney and $\mathrm{Mr} \mathbf{H}$. S. Drury for their help with the medical illustrations.

This paper is based on a communication read to the Surgical Research Society at their London meeting in January 1971.

\section{References}

Boley, S. J., Schwartz, S., Lash, J., and Sternhill, V. (1963). Reversible vascular occlusion of the colon. Surg. Gynaec. Obstet., 116, 53-60.

Civetta, J. M., and Kolodny, M. (1970). Mesenteric venous thrombosis associated with oral contraceptives. Gastroenterology, 58, 713-716.

Fishman, W. H., Green, S., and Inglis, N. I. (1963). L-phenylalanine: an organ specific, stereo-specific inhibitor of human intestinal alkaline phosphatase. Nature (Lond.), 198, 685-686.

Kilpatrick, Z. M., Silverman, J. F., Betancourt, E., Farman, J., and Lawson, J. P. (1968). Vascular occlusion of the colon and oral contraceptives. New Engl. J. Med., 278, 438-440.

Lowry, J. B., Orr, K. G., and Wade, W. G. (1969). Infarction of the small intestine associated with oral contraceptives. J. Irish med. Ass., 62, 260-262.

Marcuson, R. W., and Farman, J. A. (1971). Ischaemic disease of the colon. Proc. roy. Soc. Med., 64, 1080-1083.

Marcuson, R. W., and Tomlinson, B. (1971). In preparation.

Marston, A., Marcuson, R. W., Chapman, M., and Arthur, J. F. (1969). Experimental study of devascularisation of the colon. Gut, 10, 121-130.

Miller, D. R. (1971). Unusual focal mesenteric venous thrombosis associated with contraceptive medication: a case report. Ann. Surg., 173, 135-138.

Polk, H. C. (1966). Experimental mesenteric venous occlusion. III. Diagnosis and treatment of induced mesenteric venous thrombosis. Ann. Surg., 163, 432-444.

Reed, D. L., and Coon, W. W. (1963). Thromboembolism in patients receiving progestational drugs. New Engl. J. Med., 269, 622624.

Scott, G. B. D. (1970). Concerning the organization of thrombi. Ann. roy. Coll. Surg. Engl., 47, 335-343. 